\title{
A Compensatory Mirror Cortical Mechanism for Facial Affect Processing in Schizophrenia
}

Javier Quintana, M.D., Ph.D., Tom Davidson, B.A., Edward Kovalik, B.S. , Stephen R. Marder, M.D, and John C. Mazziotta, M.D., Ph.D.

When primates passively observe other subjects perform specific gestures or actions, premotor and motor cortical areas involved in the internal representation and actual execution of those actions exhibit neuronal activation. This mirror mechanism matches observation, representation, and execution, facilitating internal motor rehearsal, imitation, recognition of actions by others and their meanings, and social learning. Schizophrenic patients have deficits in processing affect displayed by other people's faces, which likely relates to the poor social adaptation and functioning seen in the condition. We hypothesized that, when correctly performing working-memory tasks requiring facial affect processing, schizophrenic patients would show relative increased activity in brain areas involved in social learning and in the internal representation of facial expressions when compared to controls. We used functional magnetic resonance imaging in schizophrenic patients and normal controls to detect relative changes of blood flow in cortical areas related to the representation of facial expressions while the subjects performed simple working-memory tasks with facial emotion diagrams or color circles as cues. We found that, when the task cues were facial expressions in contrast to color circles, the schizophrenic group exhibited increased activation of the face movement areas in motor and premotor cortex.

[Neuropsychopharmacology 25:915-924, 2001] (C) 2001 American College of Neuropsychopharmacology. Published by Elsevier Science Inc. All rights reserved.
KEY WORDS: Schizophrenia; Facial affect; Mirror system; Functional MRI; Working memory; Social cognition

Poor social adaptation and functioning is a hallmark of schizophrenia and may be related to the deficits at different levels of cognition that have been documented in the condition (Green 1996; Dickerson et al. 1996) . Numerous reports over the last forty years (reviewed in

From the West Los Angeles VA HCC (JQ, TD, EK, SRM), and Departments of Psychiatry (JQ, TD, EK, SRM), Neurology (JCM), Radiology (JCM), and Pharmacology (JCM), and Brain Mapping Center (JCM), School of Medicine, University of California at Los Angeles, Los Angeles, CA 90024

Address correspondence to: Dr. J. Quintana, UCLA Neuropsychioatric Institute and Hospital, Room C8-846, 760 Westwood Plaza, Los Angeles, CA 90024-1759, Tel.: 310-478-3711, ext. 41009, Fax: 310-68-4056, E-mail: xquintan@ucla.edu.

Received December 6, 2000; revised April 24, 2001; accepted May 25, 2001.

Online publication: 6/8/01 at www.acnp.org/citations/Npp060801132.
Hellewell and Whittaker 1998) indicate that patients with schizophrenia have a deficit in properly recognizing facial affect- the emotional value aspects of facial expressions (Borod et al. 1993; Streit et al. 1997; Addington and Addington 1998; Federman et al. 1998; Habel et al. 2000; Izard 1959; Dougherty et al. 1974; Muzekari and Bates 1977; Walker et al. 1980; Heimberg et al. 1992; Iscoe and Veldman 1963; Pillowski and Bassett 1980). Yet the neural correlates of these deficits have not been established. Facial affect recognition is instrumental in social learning and adaptation. Recent functional neuroimaging preliminary reports have characterized abnormalities in relative blood flow changes-a likely measure of brain activity changes (Heeger et al. 2000; Rees et al. 2000)—in specific cerebral regions of schizophrenic patients while they performed working memory or discrimination tasks involving facial affect processing (Quintana and Kovalik 1999; Hariri et al. 1999). It is difficult to determine whether changes in brain activity ob- 
served in schizophrenia represent primary deficits or secondary-even compensatory-phenomena, being that this is a chronic condition where even treatment effects have been implicated in the development of brain abnormalities. However, it is plausible to believe that, if schizophrenic patients exhibit activity increases in specific brain areas-when compared to controls-while correctly performing tasks supported by those areas, these activity increases may be the result of, or represent, cerebral compensatory efforts.

In primates, the observation of actions and gestures performed by others activates the internal representation of these actions, eliciting patterns of neuronal activity similar to those seen when the observing individual executes the same actions (Gallese et al. 1996; Hari et al. 1998; Iacoboni et al. 1999; Rizzolatti et al. 1999; Nishitani and Hari 2000). "Resonant" neural systems that match observation and execution of actions have been described in the premotor and motor cortical areas of human and non-human primates (Hari et al. 1998; Iacoboni et al. 1999; Rizzolatti et al. 1999; Nishitani and Hari 2000). Single-unit activity occurring both during behavior observation and action, especially related to hand and mouth gestures, has been recorded from neurons in the premotor cortex of macaque monkeys that supports the operation of those systems, and it has been termed mirror activity (Gallese et al. 1996; Rizzolatti et al. 1999). Resonant or mirroring systems are believed to have a role in recognizing and interpreting actions or gestures performed by others and their meanings (Gallese et al. 1996; Hari et al. 1998; Iacoboni et al. 1999; Rizzolatti et al. 1999; Nishitani and Hari 2000; Rizzolatti and Arbib 1998; Gallese and Goldman 1998), as well as in motor rehearsal (Decety et al. 1994), imitation (Iacoboni et al. 1999; Nishitani and Hari 2000), and learning (Decety et al. 1994). Thus, they support social adaptation and functioning.

We hypothesized that the correct processing of facial affect during working memory (WM) by schizophrenic patients would invoke compensatory brain mechanisms including increased activity in cerebral areas that participate in the internal representation of facial expressions for the purpose of understanding their meaning. We further hypothesized that, because of the relationship between facial affect recognition and social learning and adaptation, and the known deficits in the latter in schizophrenia, the areas involved in those particular compensatory processes should be part of brain mechanisms known to participate in social learning. Thus, if our hypotheses were correct we should detect correlates of increased neuronal activation in schizophrenic patientsrelative to controls-during correct performance of WM tasks requiring facial affect processing, particularly in brain areas related to facial affect representation and that also support social learning. An ideal candidate region for possessing those characteristics is the mirror system for facial expressions encompassing premotor-and pos- sibly also motor-areas that control and coordinate facial movements for the construction of facial expressions.

To test our hypothesis, we used functional magnetic resonance imaging (fMRI) to measure localized relative blood flow changes-a correlate of brain activity (Heeger et al. 2000; Rees et al. 2000)—in premotor and motor areas of schizophrenic patients and healthy controls. The subjects performed simple visuo-motor task paradigms where a visual cue-a color circle or a diagram of a facial expression-indicated the correct choice between, respectively, two color circles or two facial diagrams presented a few seconds later with different degrees $(50 \%$ or $100 \%$ ) of probability (see Methods section). The task rules emphasized the retention of cue information (i.e., when the correct choice could not be anticipated) or the anticipation of responses (i.e., when it could). This task design has proven useful for understanding the interactions between separate cerebral areas of distributed networks in monkeys (Quintana and Fuster 1992,1993,1999).

\section{METHODS}

\section{Subjects}

We recruited eight volunteers (six males, two females, mean age: $29.25 \pm 5.13$, all right-handed) and eight schizophrenic patients (six males, two females, mean age: $35.22 \pm 10.69$, all right-handed) whose diagnosis had been established according to DSM-IV criteria (American Psychiatric Association). The subjects received a detailed explanation of the nature and possible consequences of the study and were entered in the protocol, approved by the UCLA School of Medicine and the West Los Angeles VA Health Care Center Institutional Review Boards in compliance with the Declaration of Helsinki and the guidelines from the National Institutes of Health, after their informed consent was obtained. The control subjects had no personal or familial history of neurological or psychiatric disorders. The schizophrenic subjects were all chronic (average length of illness 8.5 years), stable outpatients, medicated with modern (i.e., atypical) antipsychotics at standard oral doses (Olanzapine 10-20 mg/ day, Risperidone 2-6 mg/day) known to have minimal effect on motor systems. None of the patients received concurrent anticholinergic medications. They were free of neurological conditions or symptoms, as revealed by a review of their past and current medical records.

\section{Experimental Task Design}

Functional MRI data were acquired while the subjects in both groups performed a series of block-design paradigms counterbalanced for order across subjects. For each paradigm, three resting blocks (black visual field) of approximately 24 seconds each were interleaved 

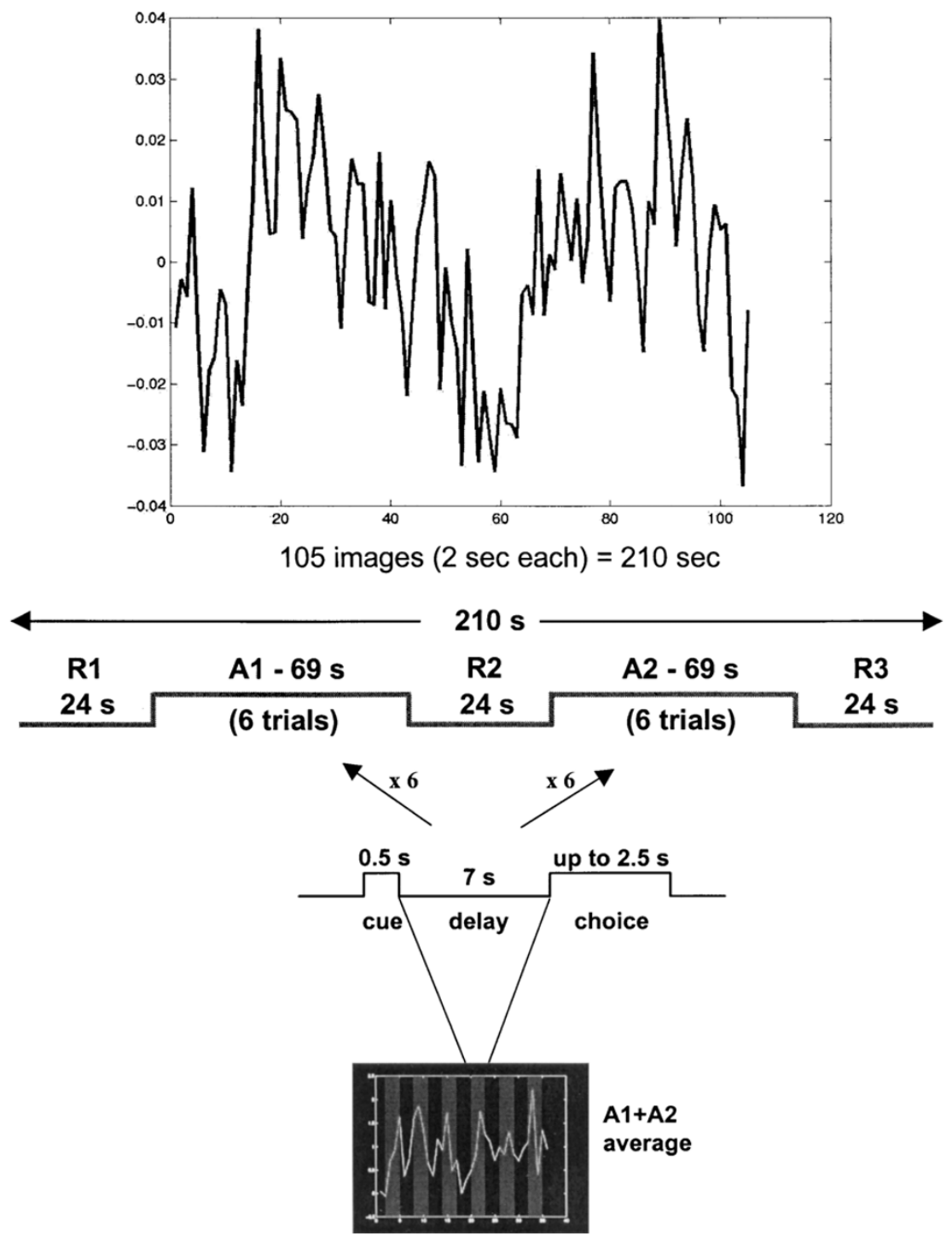

Figure 1. Temporal structure of an experimental run and trial (see Methods section). R $(1,2,3)=$ resting periods; $\mathrm{A}$ $(1,2)=$ activation periods; $\mathrm{s}=$ seconds. Each activation period contained six task trials, whose temporal structure is also depicted in a lower small diagram. Top graph: time course of changes in signal intensity throughout an entire scanning run. Observe the prominent activation during the two activation periods of the run. Bottom graph: time course of changes in signal intensity during periods of activation (average of two activation periods of a run). Observe that the relative increases in signal intensity coincide with the delay periods of the six trials. Data corresponds to left Brodmann's area 4, patients group, anticipatory task performance with facial stimuli. with two series of six trials (see Figure 1). Each trial consisted of the display, for 0.5 seconds, of a cue (see below) in the center of a black screen, a 7-sec delay (black screen only), and a choice between two stimuli similar to the cue, presented side-by-side in the middle level of a black screen background for up to 2.5 seconds. Once a choice was made according to the task contingencies explained below, the choice stimuli disappeared and the screen remained black for the rest of the 2.5 -sec period, right after which the cue for the next trial appeared. Each block of six trials lasted approximately 70 seconds, of which 3.5 seconds ( $5 \%$ of total) corresponded to cue presentations, 42 seconds ( $60 \%$ of total) to delay periods, and, on the average, $7-10$ seconds (10-15\% of total) to choice execution and $14-17$ seconds ( $20-25 \%$ of total) to intertrial periods. Thus, our design maximized the weight of delay activity in the task-related signal changes measured by fMRI. Overall, for the purpose of this study, each subject underwent four runs, each containing 12 trials.
We used two types of stimuli as cues: (a) colored circles, or (b) line drawings of faces depicting either a happy or a sad expression. The choices at the end of each trial were between two colored circles or two face diagrams presented side-by-side on a black background, respectively. For each of those two stimulus types, colors or faces, we designed two sets of cues. One set indicated with certainty the location-right or left-of the correct choice, whereas the other did not, all according to the following contingencies: a red or green cue, or a smiling or sad face, preceded a choice between red and green color circles or between smiling and sad faces respectively, presented side-by-side in random position over a black background after a 7-sec delay. The subjects were instructed to choose the response stimulusright or left-that matched the cue. Thus, in the case of this first set of cues, the predictability of the correct response side was $50 \%$ (chance level). Alternatively (second set of cues), a blue or a yellow cue preceded a 


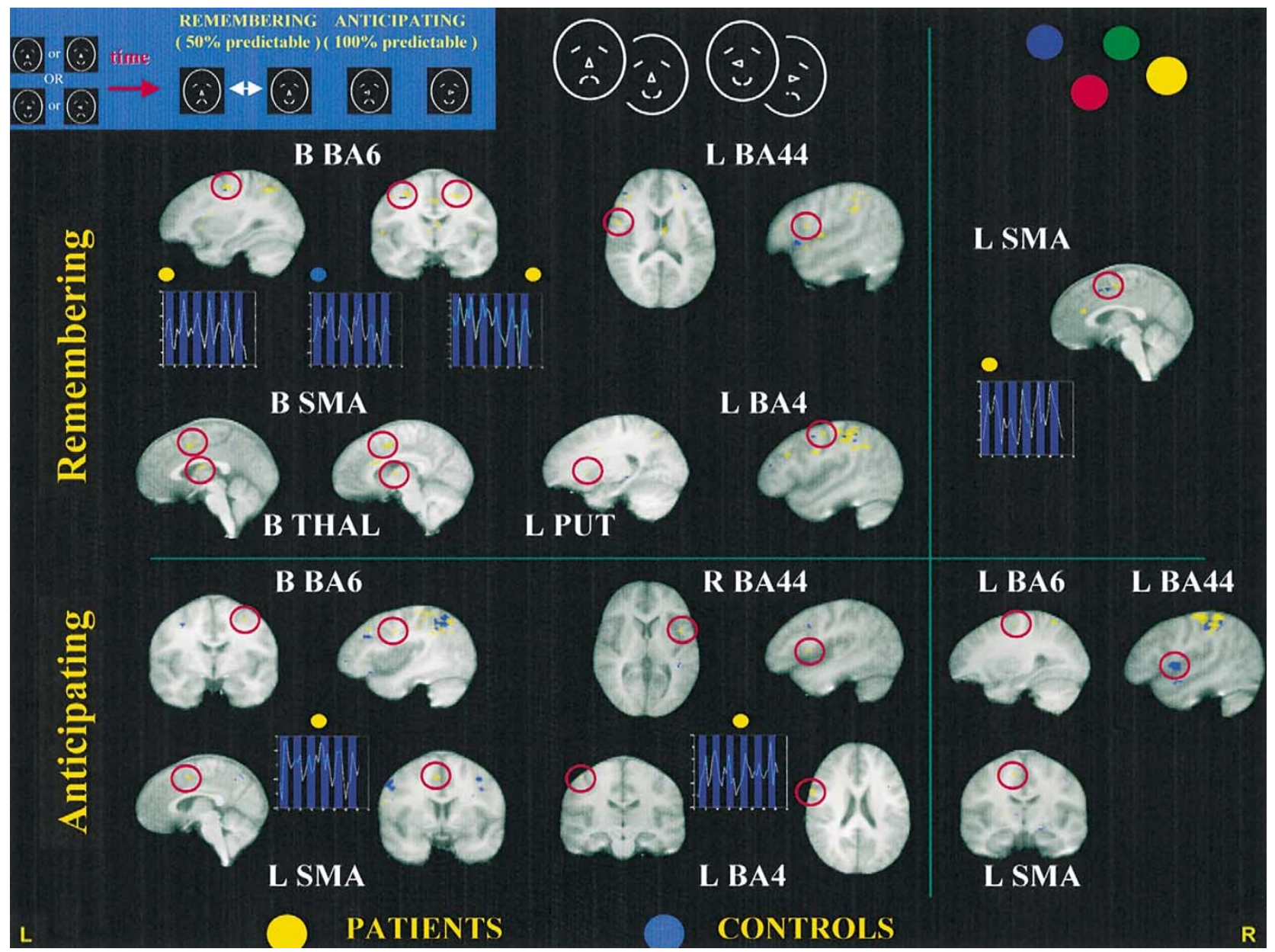

Figure 2. Cortical and subcortical areas showing statistically significant signal intensity increases related to task performance as determined by group analyses (see Methods section for details). Functional data are overlaid on Talairach-compatible templates from a brain MR atlas (Woods et al. 1998). Red rings circle significant activations, shown in yellow for the group of schizophrenic subjects and in blue for the control group (the same color code is used on top of a time series diagram indicating whether the graph represents data from patients or controls). A diagram on the top left corner represents the task trial structure, using facial stimuli as example. The figure is divided in four sectors. Activations displayed to the left of the vertical line are those seen during performance with facial expressions as stimuli, and to the right of the vertical line are those seen during color task performance. The horizontal line separates activations measured during mnemonic from those measured during anticipatory performance. Labels describe Brodmann's areas (BA= Brodmann's area; B = bilateral; L = left; $\mathrm{R}=$ right; Thal = thalamus; Put = Putamen; SMA = supplementary motor area). See Table 1 for anatomical description, Talairach coordinates of each activation's peak, cluster extents and statistical significance scores. For some activations of special relevance to the overall results, the corresponding time series of the signal variations of the most significant voxel over the task activation periods is displayed as an average of all runs from all subjects of the particular group and task (diagram insert). Periodic blue bands on the time series graphs represent delay (memory) periods of the task. In all of the time series the activity oscillates periodically six times, the exact number of trials in each activation period and thus of averaged delay periods represented in each graph. The relative position of the bands is precise with respect to the activity changes, because the activation paradigm design automatically compensated for any response time differences between trials or subjects, by adding a variable waiting period until 2.5 seconds after the end of the delay and the onset of the response period.

choice between two side-by-side white circles. And a smiling or a sad face, whose nose pointed at the right or left of the subject's visual field, preceded a choice between a smiling and a sad face (also presented sideby-side, the one on the left with its nose pointing to the left of the subject, the one on the right with a nose pointing to the right). The subjects had been instructed before the scanning session that after a blue color, the correct response was always the left white circle, and after a yellow color, the right white circle. They had also been instructed that the direction where the nose of the face cue was pointing always determined the correct response between the two face choices, left or right. Hence, the predictability of correct response here was 100\%, al- 
though the subjects could not execute a response until the two choices appeared following the cue. Each trial series randomly used two cues of the same type (color or face) and of the same set (i.e., same level of response predictability - certain or uncertain correct choice side) (e.g., blue and yellow, red and green, smiling and sad faces, faces with right- and left-pointing noses).

\section{Imaging Protocols}

Subjects were fitted with magnetic-compatible goggles with built-in LCD screens on which the computer-programmed stimulation paradigms were displayed. They responded via button-pressing (left or right) on a mouse device with minimal movement of two fingers of the dominant (right) hand. Performance data was recorded by means of microcomputer control and statistically analyzed using a logistic regression model for response correctness and a multiple regression model for reaction time. Images were acquired using a GE 3T scanner with an echo-planar imaging upgrade. First, we obtained coplanar structural images consisting of 26 slices $(4 \mathrm{~mm}$ thick, $1 \mathrm{~mm}$ gap) that covered the entire brain volume $(\mathrm{TR} / \mathrm{TE}=4000 / 54 \mathrm{msec})$. Then we acquired gradient echo (GRE) functional images covering 14 slices $(4 \mathrm{~mm}$ thick, $1 \mathrm{~mm}$ gap), beginning near the middle of the temporal lobes and moving upward (TR/TE $=2000 / 45$ $\mathrm{msec}$ ). Because of this volume limitation, areas such as amygdala and cerebellum that may play a role in processing affect and memory were not explored in this study. For each subject, images were first realigned to correct for head motion (Woods et al. 1998), then normalized into a standard stereotaxic space. We smoothed the imaging data using a Gaussian filter set at $6 \mathrm{~mm}$ fullwidth at half-maximum to minimize noise and residual differences in gyral anatomy. Finally, a group analysis of the pre-processed images was completed using a general linear model with a delayed box-car reference function (SPM 99) (Friston et al. 1994), comparing average signal intensity during trial-performance periods with the average signal intensity during resting periods immediately before and after each series of trials. Additionally, we performed a first-level conjunction analysis (Friston et al. 1999) with implicit modeling of resting periods, contrasting the activations detected in both groups. Because of the described effects of motion on individual and group data (Hajnal et al. 1994; Friston et al. 1996), we carefully investigated the presence of motion at the individual and group level and concluded that only an insignificant drift of less than $0.4 \mathrm{~mm}$, progressive and unrelated to task periods, was present in our samples. When a significant activation in the group data was identified, we investigated its variability in voxel intensity, cluster extent, and time-course across all subjects within our design matrix, in which intersubject variability is normalized via proportional scal- ing. We found the variance in voxels of interest to be smooth across subjects and with few outliers, denoting a tight distribution of voxel intensity as it appears after scaling to whole brain mean signal of the group.

\section{RESULTS}

Both subject groups in our study, patients and controls, performed the tasks at statistically similar levels of accuracy, as determined by logistic (for response correctness, $p=.09$ ) and multiple (for reaction time, $p=.49$ ) regression model analyses of the performance data. Given the block paradigm design used, we could notunfortunately-analyze activation patterns during trials with incorrect response, but the overall error rate was only $13.8 \%$. The imaging data analyses revealed discrete, statistically significant, relative blood flow changes in areas of the motor and premotor cortex, as well as in Broca's area and subcortical nuclei (Figure 2, Table 1). Time series analyses confirmed that the relative fMRI signal intensity changes measured were related to task performance (Figures 1 and 2). We observed differences in activation patterns within and between groups mostly as a result of task variations in type of information (i.e., color circles or facial diagrams depicting an emotion). Overall, among both patients and controls, more activation in cortical motor systems was seen when the information needed for correct task performance was of emotional (i.e., facial expressions) than when it was of logical (i.e., colors) nature. Specific differences between schizophrenic patients and control subjects supporting our hypothesis occurred at the levels of premotor and motor cortex (in areas extending over the face, mouth, and lips representation), supplementary motor area (SMA), and Broca's area (Figure 2, Table 1).

During task performance requiring the processing of facial expressions depicted by a diagram, either by retaining facial information in WM or by anticipating responses on the basis of such information, schizophrenic subjects showed a pattern of bilateral increases in relative blood flow in Brodmann's premotor area 6, and unilateral (left, dominant) increases in area 4 and Broca's area (44 of Brodmann's). They also showed relative blood flow increases in SMA (bilaterally), thalamus (bilaterally, anterior and mediodorsal nuclei) and in left putamen during mnemonic task performance with facial expressions as cues, and left SMA and right Brodmann's area 44 during the anticipatory task performance with facial diagrams. On the other hand, also when using these diagrams as cues for task performance, healthy controls showed only premotor (Brodmann's area 6) activation in the left hemisphere during the mnemonic task and predominantly in the right premotor cortex during the anticipatory task, with a minimal acti- 


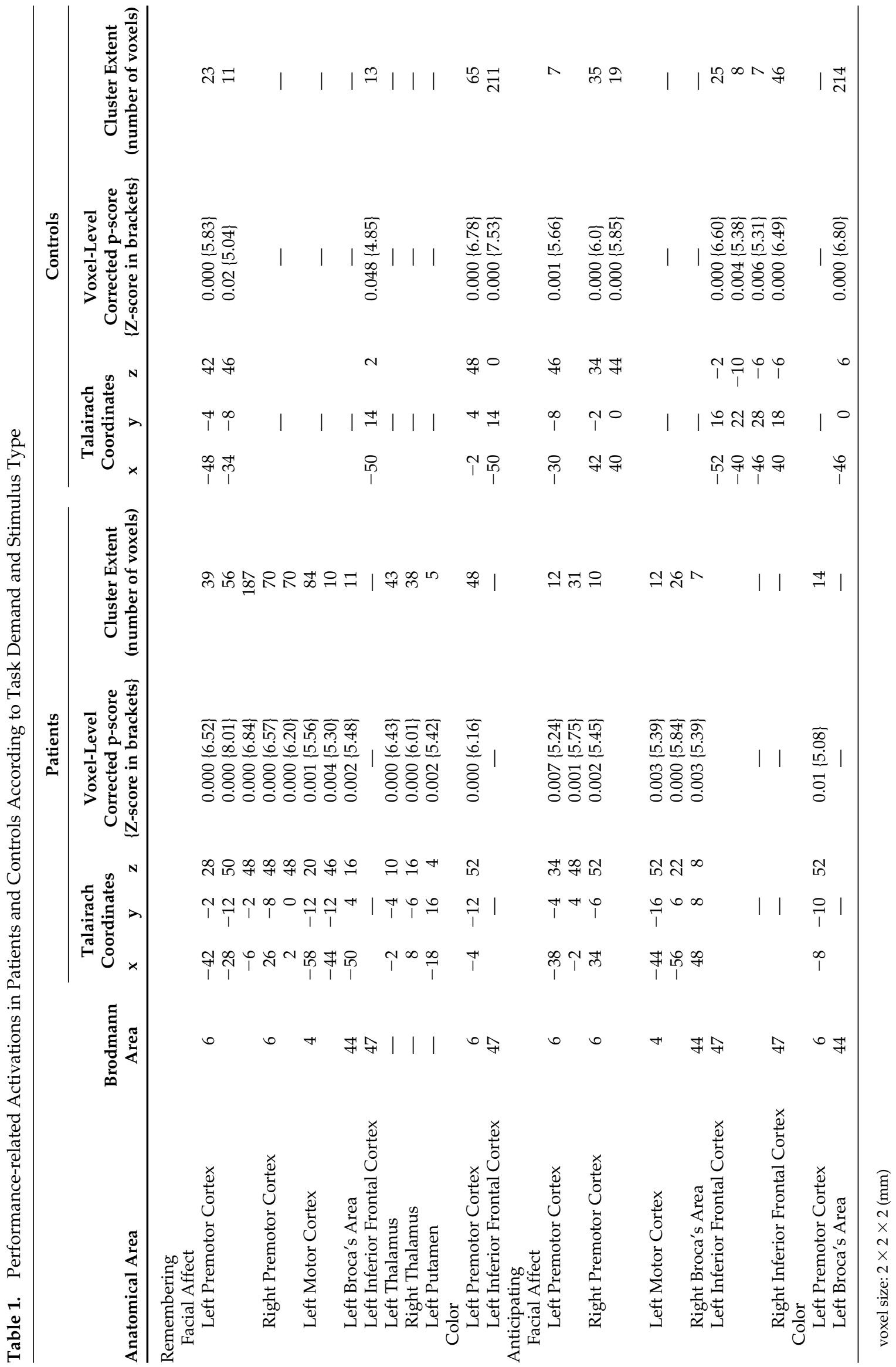


vation also on the left side in this latter case (Figure 2, Table 1). Finally, the control group showed activation of area 47 of Brodmann's (inferior frontal cortex, IFC) that was absent in the patients group.

When the information needed to perform the task was color, however, the activation pattern differences between the two subject groups was no longer observed. We found limited activation of the left SMA - in both groups during retention of color information and only among the schizophrenic patients during anticipation of responses based on color information. In this later case, a significant activation of the left Broca's area, as well as of Brodmann's area 47 in the IFC, were seen solely in the control group (Figure 2, Table 1). Neither group showed area 47 activation in any other performance situation using color cues.

\section{DISCUSSION}

Our findings indicate that, during correct performance of decision-making tasks that demand processing facial expressions in working memory, motor cortical areas involved in the representation and execution of facial movements responsible for those expressions become significantly activated in patients with schizophrenia but not in control subjects. At the same time, areas of the IFC connected to limbic structures appear activated in normal controls only. These differences occur in spite of changes on the temporal dynamics of the tasks (i.e., remembering information or anticipating responses) as long as the information involves facial emotional expressions.

Two questions at least come to mind when interpreting our results: could they be the product of artifacts (e.g., subject's motion)? Can they be considered as indication of abnormal activation, perhaps compensatory, of a mirror system for facial expressions in schizophrenia? Regarding the first issue, both groups of subjects were required to perform the tasks under similarly strict motionless conditions, and asked to respond in an equally controlled manner. No significantly increased head motion artifacts, which could result from facial movements, were observed among the schizophrenic patients or detected during data analyses. The subjects were observed during the initial brief period of acquaintance with the task structure and rules, and during the brief practice they were allowed before their placement on the scanner bed; no particular facial movement was observed that could be responsible for the activation seen in the results. We did not obtain electromyographic records from the subjects during performance, a point that we could address in future studies in spite of technical difficulties. However, the fact that motor and premotor cortex activation in our study was seen only during the task performance guided by facial expression informa- tion further suggests that the phenomenon is not a reflection of subjects' movements. The same argument applies to any putative medication effects on the results.

Correlating motor or premotor cortical activity detected with fMRI to any particular body structure is imprecise in the absence of corroborating evidence from concurrent movements. Although we did not obtain specific motor cortical maps for any of our subjects, the tridimensional coordinates of the activation areas here described do not disagree with those reported for the face, mouth and lips areas in somatotopic studies of the motor-premotor body representation (Grafton et al. 1991; Lotze et al. 2000), particularly when accounting for factors such as overlapping in cortical maps and the impact of averaging described in these and other studies (Grafton et al. 1993). Furthermore, the crucial information necessary to correctly perform our task was located on the mouth-lips area of the face diagrams used as cues. Thus, the observed changes in task-related signal intensity occurred during observation of a diagram depicting an action or gesture, and in cortical areas where such action or gesture is represented for a putative motor output (i.e., there is an implicit matching between observation and execution). Whereas, strictly speaking, we should not equal those changes to mirror activity, for a canonical definition of mirror activity requires a complete match between an action observation and its actual execution, we can interpret them as enhanced internal representation of the observed facial expressions. Another suggested interpretation is the presence of positive symptoms in the schizophrenic subjects. However, we did not find parallel differential activations in somatosensory cortex (which is an important component of the neuronal circuitry thought to be dedicated to the internal representation of emotions in the theory of mind), nor in visual cortex (which has been shown to be involved during active production of visual hallucinations and visual imagery). Finally, we did not detect statistical differences in performance levels between the groups or in activation of the visual cortex that would indicate an effect of cue complexity in the results.

In the case of the observed primary motor cortex activation, it is conceivable that an expansion of premotor activity might facilitate changes in corresponding primary motor areas. However, the co-activation of area 4, contralateral to the performing hand, and area 6 bilaterally has been reported in studies of execution-simulation matching (Roth et al. 1996). Moreover, the activation of Broca's area has been suggested as a crucial element in imitation and mirror systems in humans (Iacoboni et al. 1999). Hence, a selective co-activation of the representation of facial movements on these three cortical elements (i.e., left area 4, bilateral area 6, and Broca's area) in the group of patients and only during 
processing of information conveyed by observed facial gestures strongly suggests that an enhanced internal representation of the motor aspects of facial expressions helps subjects with schizophrenia to process the emotional meaning of these expressions. The lack of significant differences in overall performance or reaction time between controls and patients supports our hypothesis of a compensatory mechanism. We cannot directly correlate the abnormalities detected to any behavioral deficits in facial affect discrimination or processing in our subjects, who nonetheless exhibited concurrent activation deficits in IFC. However, we feel that our results can be interpreted in the context of the well-established presence of deficits in facial information processing in schizophrenic patients at large. Although such processing has been shown to involve the right somatosensory and parietal cortex (Haxby et al. 2000), the laterality seen in our results reflects the pattern described for mirror systems, and thus must be interpreted in the context of these systems.

Single-unit recording studies in monkeys and functional imaging studies in healthy human volunteers suggest that the motor systems can show activation in the absence of motor output, similar to that found during actual motor acts (Gallese et al. 1996; Hari et al. 1998; Iacoboni et al. 1999; Nishitani and Hari 2000; Roth et al. 1996; Jeannerod and Frak 1999). There is evidence that imaging a movement is associated with activation of the motor areas that would be normally responsible for generating such movement (Jeannerod and Frak 1999; Schnitzler et al. 1997). Resonant or mirroring systems that match execution and observation of an action have been described that may help in the understanding of the behaviors, actions, and intentions of other individuals in society (Gallese et al. 1996; Hari et al. 1998; Iacoboni et al. 1999; Rizzolatti et al. 1999; Nishitani and Hari 2000; Rizzolatti and Arbib 1998; Gallese and Goldman 1998; Decety et al. 1994). These systems are capable of representing "motor ideas" such as facial movements that convey emotional or intentional states of importance in social communication (Fadiga et al. 2000) and thus appear crucial for social functioning and adaptation, deficits of which are well known in schizophrenia.

Our main objective was to characterize abnormal brain mechanisms related to the well known social functioning deficits in schizophrenia, and that link social learning and adaptation with facial affect processing at the cerebral level. In our tasks, we wanted to emphasize the use-in WM-of information relevant for facial affect processing, because the known WM deficits in schizophrenic patients (Jeste et al. 1996) might enhance related compensatory activity under conditions of correct performance. We thus expected to see abnormal activity in areas involved in resonance or mirroring phenomena rather than in sensory areas related to instant (i.e., perceptual, non-mnemonic) discrimination of facial features such as the fusiform gyrus (Halgren et al.
2000) or the superior temporal sulcus or inferior frontal cortex (Nakamura et al. 1999; Haxby et al. 2000; Allison et al. 2000), which are the focus of other studies. A functional dissociation between areas involved in facial information processing has been suggested before when memory-encoding is involved (Decety et al. 1997). Characterizing compensatory mechanisms and their potential modulation is important for the social rehabilitation of schizophrenic patients. But it is well known that schizophrenia involves frontal function deficits (Jeste et al. 1996; Ingvar and Franzen 1974; Weinberger et al. 1986; Callicott et al. 1998; Heckers et al. 1999); in fact, prefrontal and inferior frontal abnormalities we will report elsewhere co-occurred in our patient's sample. What then is the relationship between the mechanisms described here and the prefrontal abnormalities observed in schizophrenia?

In normal individuals, the prefrontal cortex hosts mechanisms supporting cognitive processing and motor control, and thus the planning and execution of goal oriented behaviors in response to visual information (Fuster 1989). Working memory, attention, planning, and other executive prefrontal functions are crucial in preparing for and performing willed responses. Ultimately, however, the prefrontal cortex exercises its executive role by means of its control on motor systems activity and output. The prefrontal inhibition of unwanted, inappropriate, or untimely motor behavior is specially important for the unobstructed generation of adaptive behavior, and prefrontal gating mechanisms for perceptual inputs and motor outputs are well established (Fuster 1989; Hauser 1999; Konishi et al. 1999; Knight et al. 1999; Weinberger et al. 1983; Andreasen et al. 1986). Frontal lobe patients are known to produce uncontrolled behaviors imitating simple actions that other individuals perform in their presence (L'Hermitte et al. 1972). Frontal lobe pathology has been documented in schizophrenia (Jeste et al. 1996; Weinberger et al. 1986), and flawed frontal gating mechanisms have been hypothetically associated to the perceptual and information-processing deficits seen in this condition (Braff et al. 1995; Nuechterlein and Dawson 1984). Schizophrenic patients show motor abnormalities such as grimacing, echopraxia, posturing, stereotypy, echolalia and others in speech production, which could be the result of prefrontal gating failures. Hence, as these lines of evidence point out, it is likely that a prefrontal inhibitory deficit of motor system activity exists in schizophrenic patients. The mirror-like premotor and motor increased compensatory activity we describe here could be facilitated by such a prefrontal deficit, particularly when processing of facial affect information relevant for social functioning is required that demands additional resources. Patients with schizophrenia exhibit a number of sensory and perceptual processing deficits (Braff et al. 1995; Nuechterlein and Dawson 1984) likely contrib- 
uting to prefrontal-based perception-action cycle abnormalities. Among those, deficits specifically related to facial affect discrimination may involve cerebral sensory systems such as the superior temporal sulcus, the fusiform gyrus, and the inferior frontal cortex, as some studies indicate (Hariri et al. 1999; Nakamura et al. 1999; Haxby et al. 2000; Allison et al. 2000). Yet the specific association of increased mirror-like representational mechanisms described in our patients with cues of obvious social value stresses the relationship between prefrontal deficits and poor social adaptation in schizophrenia. The fact that patients with schizophrenia appear to use relatively unnatural-when compared to normal individuals-brain mechanisms to interpret and properly use cues instrumental for social functioning may contribute to understanding the difficulties that these individuals feel and manifest in social situations.

\section{ACKNOWLEDGMENTS}

We thank Dr. A. Kopelowicz (San Fernando Mental Health Center, Los Angeles Department of Mental Health) and Drs. W. Wirshing, D. Ames-Wirshing, and Scott Saunders (Schizophrenic Research Clinic, West Los Angeles VA Health Care Center) for generously agreeing to the recruitment of subjects from their respective clinics. We also thank Dr. Marco Iacoboni for his generosity and his time in reviewing the manuscript; his suggestions are greatly appreciated. Supported by a Department of Veteran Affairs (Office of Research and Development, Medical Research Service) Advanced Research Scientist Career Development Award, and by a NARSAD Staglin Young Investigator Award to J.Q., as well as by contributions from the VA's VISN-22 MIRECC, the Brain Mapping Medical Research Organization, the Brain Mapping Support Foundation, the Pierson-Lovelace Foundation, the Ahmanson Foundation, the Tamkim Foundation, the Jennifer-Jones Simon Foundation, the Capital Group Companies Charitable Foundation, the Robson Family, the Northstar Fund, and a National Center for Research Resources grant RR12169.

\section{REFERENCES}

Addington J, Addington D (1998): Facial affect recognition and information processing in schizophrenia and bipolar disorder. Schizophr Res 32:171-181

Allison T, Puce A, McCarthy G (2000): Social perception from visual cues: role of the STS region. Trends Cogn Sci 4:267-278

Andreasen N, Narsallah HA, Dunn V, Olson SC, Grove WM, Ehrhardt JC, Coffman JA, Crossett JHW (1986): Structural abnormalities in the frontal system in schizophrenia. Arch Gen Psychiatry 43:136-144

Borod JC, Martin CC, Alpert M, Brozgold A, Welkowitz J (1993): Perception of facial emotion in schizophrenic and right brain-damaged patients. J Nerv Ment Dis 181:494-502

Braff DL, Swerdlow NR, Geyer MA (1995): Gating and habituation deficits in the schizophrenia disorders. Clin Neurosci 3:131-139
Callicott JH, Ramsey NF, Tallent K, Bertolino A, Knable MB, Coppola R, Goldberg T, Van Gelderen P, Mattay VS, Frank JA, Moonen CT, Weinberger DR (1998): Functional magnetic resonance imaging brain mapping in psychiatry: methodological issues illustrated in a study of working memory in schizophrenia. Neuropsychopharmacology 18:186-196

Decety J, Grezes J, Costes N, Perani D, Jeannerod M, Procyk E, Grassi F, Fazio F (1997): Brain activity during observation of actions. Influence of action content and subject's strategy. Brain 120:1763-1777

Decety J, Perani D, Jeannerod M, Bettinardi V, Tadary B, Woods R, Mazziotta JC, Fazio F (1994): Mapping motor representations with positron emission tomography. Nature 371(6498):600-602

Dickerson F, Boronow JJ, Ringel N, Parente F (1996): Neurocognitive deficits and social functioning in outpatients with schizophrenia. Schizophr Res 21:75-83

Dougherty F, Bartlett E, Izard C (1974): Response of schizophrenics to expressions of the fundamental emotions. J Clin Psychol 30:243-246

Fadiga L, Fogassi L, Gallese V, Rizzolatti G (2000): Visuomotor neurons: ambiguity of the discharge or 'motor' perception? Int J Psychophysiol 35:165-177

Federman EJ, Drebing CE, Zaref JI, Oepen G (1998): Processing and recognition of facial affect in schizophrenia. Percept Mot Skills 87:484-486

Friston KJ, Jezzard P, Turner R (1994): Analysis of functional MRI time series. Human Brain Mapp 1:153-171

Friston KJ, Williams S, Howard R, Frackowiak, RSJ, Turner $R$ (1996): Movement related effects in fMRI time-series. Mag Res Med 35:346-355

Friston KJ, Holmes AP, Price CJ, Buchel C, Worsley KJ (1999): Multisubject fMRI studies and conjunction analyses. Neuroimage 9:385-396

Fuster JM (1989): The Prefrontal Cortex: Anatomy, Physiology, and Neuropsychology of the Frontal Lobe, 2nd ed. New York, Raven Press

Gallese V, Fadiga L, Fogassi L, Rizzolatti G (1996): Action recognition in the premotor cortex. Brain 119:593-609

Gallese V, Goldman A (1998): Mirror neurons and the simulation theory of mind-reading. Trends Cogn Sci. 2:493-501

Grafton ST, Woods RP, Mazziotta JC, Phelps ME (1991): Somatotopic mapping of the primary motor cortex in humans: activation studies with cerebral blood flow and positron emission tomography. J Neurophysiol 66:735-743

Grafton ST, Woods RP, Mazziotta JC (1993): Within-arm somatotopy in human motor areas determined by positron emission tomography imaging of cerebral blood flow. Exp Brain Res 95:172-176

Green MF (1996): What are the functional consequences of neurocognitive deficits in schizophrenia? Am J Psychiatry 153:321-330

Habel U, Gur RC, Mandal MK, Salloum JB, Gur RE, Schneider F (2000): Emotional processing in schizophrenia across cultures: standardized measures of discrimination and experience. Schizophr Res 42:57-66

Hajnal JV, Myers R, Oatridge A, Schwieso JE, Young IR, Bydder GM (1994): Artifacts due to stimulus correlated motion in functional imaging of the brain. Mag Res Imaging 31:283-291 
Halgren E, Raij T, Marinkovic K, Jousmaki V, Hari R (2000): Cognitive response profile of the human fusiform face area as determined by MEG. Cereb Cortex 10:69-81

Hari R, Forss N, Avikainen S, Kirveskari E, Salenius S, Rizzolatti G (1998): Activation of human primary motor cortex during action observation: a neuromagnetic study. Proc Natl Acad Sci 95:15061-5

Hariri A, Quintana J, Kovalik E, Bookheimer S (1999): Processing of facial affect in schizophrenics: an fMRI study. Neuroimage 9:647

Hauser MD (1999): Perseveration, inhibition and the prefrontal cortex: a new look. Curr Opin Neurobiol 9: 214-222

Haxby JV, Hoffman EA, Gobbini MI (2000): The distributed human neural system for face perception. Trends Cogn Sci 4:223-233

Heckers S, Goff D, Schacter DL, Savage CR, Fischman AJ, Alpert NM, Rauch S (1999): Functional imaging of memory retrieval in deficit vs nondeficit schizophrenia. Arch Gen Psychiatr 56:1117-1123

Heeger DJ, Huk AC, Geisler WS, Albrecht DG (2000): Spikes versus BOLD: what does neuroimaging tell us about neuronal activity? Nature Neurosci 3:631-633

Heimberg C, Gur RE, Erwin RJ, Shtasel DL, Gur RC (1992): Facial emotion discrimination: III. Behavioral findings in schizophrenia. Psychiatry Res 42:253-265

Hellewell JSE, Whittaker JF (1998): Affect perception and social knowledge in schizophrenia. In Mueser KT, Tarrier N (eds.), Handbook of social functioning in schizophrenia. Boston, Allyn \& Bacon, pp 197-212

Iacoboni M, Woods RP, Brass M, Bekkering H, Mazziotta JC, Rizzolatti G (1999): Cortical mechanisms of human imitation. Science 286:2526-2528

Ingvar DH, Franzen G (1974): Abnormalities of cerebral blood flow distribution in patients with chronic schizophrenia. Acta Psychiatr Scand 50:425-462

Iscoe I, Veldman DJ (1963): Perception of an emotional continuum by schizophrenics, normal adults and children. J Clin Psychol 19:272-276

Izard C (1959): Paranoid schizophrenic and normal subjects' perception of photographs of human faces. J Consult Psychol 23:119-124

Jeannerod M, Frak V (1999): Mental imaging of motor activity in humans. Curr Opin Neurobiol 9:735-739

Jeste DV, Galasko D, Corey-Bloom J, Walens S, Granholm E (1996): Neuropsychiatric Aspects of the Schizophrenias. In Fogel BS, Schiffer RB, Rao SM (eds), Neuropsychiatry. Baltimore, Williams \& Wilkins, pp 325-344

Knight RT, Staines WR, Swick D, Chao LL (1999): Prefrontal cortex regulates inhibition and excitation in distributed neural networks. Acta Psychol 101:159-178

Konishi S, Nakajima K, Uchida I, Kikyo H, Kameyama M, Miyashita Y (1999): Common Inhibitory mechanism in human inferior prefrontal cortex revealed by eventrelated functional MRI. Brain 122:981-991

L'Hermitte F, Deroulsne J, Signoret JL (1972): [Neuropsychological analysis of the frontal syndrome]. Rev Neurol 127:415-440

Lotze M, Erb M, Flor H, Huelsmann E, Godde B, Grodd W (2000): fMRI evaluation of somatotopic representation in human primary motor cortex. Neuroimage 11:473-481
Muzekari LH, Bates ME (1977): Judgment of emotion among chronic schizophrenics. J Clin Psychol 33:662-666

Nakamura K, Kawashima R, Ito K, Sugiura M, Kato T, Nakamura A, Hatano K, Nagumo S, Kubota K, Fukuda H, Kojima S (1999): Activation of the right inferior frontal cortex during assessment of facial emotion. J Neurophysiol 82:1610-1614

Nishitani N, Hari R (2000): Temporal dynamics of cortical representation for action. Proc Natl Acad Sci 97: 913-918

Nuechterlein KH, Dawson ME (1984): Information processing and attentional functioning in the development course of schizophrenic disorders. Schizophrenia Bull 10:160-203

Pillowski I, Bassett D (1980): Schizophrenia and the response to facial emotions. Compr Psychiatry 21:236-244

Quintana J, Fuster JM (1992): Mnemonic and predictive functions of cortical neurons in a memory task. Neuroreport 3:721-724

Quintana J, Fuster JM (1993): Spatial and temporal factors in the role of prefrontal and parietal cortex in visuo-motor integration. Cerebral Cortex 3:122-132

Quintana J, Fuster JM (1999): From perception to action: temporal integrative functions of prefrontal and parietal neurons. Cerebral Cortex 9:213-221

Quintana J, Kovalik E (1999): Differences in prefrontal and parietal association cortex activation between controls and schizophrenic patients during decision-making working memory tasks. Soc Neurosci Abs 25:19

Rees G, Friston K, Koch C (2000): A direct quantitative relationship between the functional properties of human and macaque V5. Nature Neurosci 3:716-723

Rizzolatti G, Arbib MA (1998): Language within our grasp. Trends Neurosci 21:188-194

Rizzolatti G, Fadiga L, Fogassi L, Gallese V (1999): Resonance behaviors and mirror neurons. Arch Ital Biol 137:85-100

Roth M, Decety J, Raybaudi M, Massarelli R, Delon-Martin C, Segebarth C, Morand S, Gemignani A, Decorps M, Jeannerod M (1996): Possible involvement of primary motor cortex in mentally simulated movement: a functional magnetic resonance imaging study. Neuroreport 7:1280-1284

Schnitzler A, Salenius S, Salmelin R, Jousmaki V, Hari R (1997): Involvement of primary motor cortex in motor imagery: a neuromagnetic study. Neuroimage 6:201-208

Streit M, Wolwer W, Gaebel W (1997): Facial-affect recognition and visual scanning behaviour in the course of schizophrenia. Schizophr Res 24:311-317

Walker E, Marwit SJ, Emory E (1980): A cross-sectional study of emotion recognition in schizophrenics. J Abnorm Psychol 89:428-436

Weinberger DR, Berman KF, Zec RF (1986): Physiologic dysfunction of dorsolateral prefrontal cortex in schizophrenia. Arch Gen Psychiatry 43:114-124

Weinberger DR, Wagner RL, Wyatt RJ (1983): Neuropathological studies of schizophrenia: a selective review. Schizophr Bull 9:193-212

Woods RP, Grafton ST, Holmes CJ, Cherry SR, Mazziotta JC (1998): Automated image registration: 1. General methods and intrasubject, intramodality validation. J Comp Assist Tomography 22:139-152 